REVISTA DE DERECHO UNED, NÚM. 23, 2018

\title{
LA FINANCIACIÓN POSTCONCURSAL DEL DEUDOR
}

\author{
THE POSTBANKRUPTCY FINANCING OF THE DEBTOR
}

\author{
CÉSAR GILo GÓMEZ
}

Doctor en Derecho. Universidad de Salamanca. Abogado

Resumen: El legislador español ha centrado sus esfuerzos en los últimos años en desarrollar mecanismos que permitiesen evitar la declaración de concurso dotando a la Ley Concursal de fórmulas preventivas dirigidas a ese fin. Sin embargo, se ha despreocupado de otorgar al deudor las herramientas necesarias que le permitiesen salir de la situación de insolvencia una vez que el concurso ya es declarado.

Y es que para remediar referida situación una vez que el deudor se encuentra inmerso en el procedimiento concursal, la entrada de nueva financiación en el patrimonio del deudor insolvente se convierte en una cuestión fundamental, ya que sólo con la pertinente inyección de liquidez, éste podrá mantener su actividad económica, la cual es el vehículo que debe permitirle generar la riqueza necesaria para cumplir con sus obligaciones y salir de la situación de insolvencia en la que se encuentra.

Por esta razón, en el presente trabajo se estudia la situación actual que el estado de la cuestión presenta, analizando las posibilidades que el texto normativo permite al deudor para mantener su actividad económica, así como las reformas que serían necesarias para estimular la entrada de nueva financiación en el patrimonio del deudor insolvente.

Palabras clave: Ley Concursal, deudor, financiación.

Abstract: The Spanish legislator has centred his efforts in the years last to develop mechanisms that were allowing to avoid the 
declaration of bankruptcy providing to the Bankruptcy Act of preventive formulae directed this purpose. Nevertheless, there is the unconcerned one of granting to the debtor the necessary toolsthat were allowing him to go out of the situation of insolvency as soon as the contest already is declared.

And it is that to remedy above mentioned situation as soon as the debtor is inmmersed in the bankruptcy proceeding the entry of new financing in the heritage of the insolvent debtor turns into a fundamental question, since only with the pertinent injection of liquidity, this one will be able to support his economic activity, which is the vehicle that must allow him to generate the necessary wealth to expire with his obligations and to go out of the situation of insolvency in the one that is.

For this reason, in the present work there is studied the current situation that the state of the question presents, analyzing the possibilities that the normative text allows to the debtor to support his economic activity, as well as the reforms that would be necessary to stimulate the entry of new financing in the heritage of the insolvent debtor.

Keywords: Bankruptcy Act, debtor, financing.

Recepción original: 23/05/2018

Aceptación original: 15/10/2018

Sumario: I. Introducción.-II. Entidades de crédito y financiación postconcursal.- III. Administración concursal y financiación postconcursal-- IV. Acreedores y financiación postconcursal.V. Garantías reales y financiación postconcursal.-VI. La financiación postconcursal en el derecho comparado.-VII. Conclusiones.

\section{INTRODUCCIÓN}

Los 14 años que han transcurrido desde la entrada en vigor de la Ley 22/2003, de 9 de julio, Concursal —en adelante LC - han puesto de manifiesto que la inmensa mayoría de sociedades que acuden al procedimiento concursal terminan desapareciendo por medio de la liquidación de sus activos.

Por esta razón, desde el año 2009 el legislador concursal ha introducido reformas para paliar esta situación, fomentando la negociación en la fase preconcursal para evitar con ello que el concurso de una sociedad sea finalmente declarado. La premisa es clara: si la 
mayoría de las empresas que entran en concurso se ven abocadas a la liquidación, evitemos que las mismas acudan a él y superen sus problemas antes de ser intervenidas judicialmente.

La iniciativa del legislador adolecía del defecto de centrar exclusivamente los esfuerzos en la fase preconcursal, pretiriendo la fase postconcursal o mejor dicho, la fase propiamente concursal.

Y es aquí donde entendemos que existe una de las más importantes fallas de nuestro sistema de insolvencia: carecer de un adecuado procedimiento de financiación postconcursal que permita al deudor declarado en concurso de acreedores recibir fondos para poder hacer frente a los gastos necesarios que la continuación de su actividad económica supone.

No ignoramos que el apartado $\mathrm{V}$ de la Exposición de Motivos de la Ley 38/2011, de 10 de octubre, de reforma de la LC prevé la consideración como créditos contra la masa de los créditos por financiación nacidos tras la aprobación judicial del convenio en caso de apertura posterior de la fase de liquidación. Sin embargo, aun siendo cierto que el artículo 84.2.11 LC recoge la calificación como créditos contra la masa de estos créditos, referido precepto se remite al artículo 100.5 LC, el cual establece el pago de mencionados créditos en los términos fijados en el convenio. Es decir, que en la Exposición de Motivos se anuncia el blindaje de este tipo de créditos, se reafirma su consideración como crédito contra la masa en el artículo 84.2.11 LC pero finalmente se deja en el artículo 100.5 LC a la autonomía de las partes la manera en la que se va a hacer frente a los mismos, por lo que sólo en caso de que se abra la fase de liquidación podrá asegurarse que estos créditos tendrán la consideración de créditos contra la masa ${ }^{1}$.

Esta desafortunada remisión se debe a que se ha mantenido la redacción originaria del artículo 100.5 LC a pesar de la reforma introducida por la Ley 38/2011, de tal modo que actualmente coexiste la consideración de los créditos concedidos tras la aprobación judicial del convenio en caso de apertura posterior de la fase de liquidación como créditos contra la masa a tenor del artículo 84.2.11 LC con la autonomía de la voluntad conforme a convenio prevista en el 100.5 LC para el supuesto de que la fase de liquidación no se llegase a abrir.

1 Tanto el principal del crédito como sus intereses. Así HERNÁNDEZ SAINZ, E., «Los créditos contra la masa» en GARCÍA-CRUCES, J.A., Jurisprudencia y Concurso, Tirant lo Blanch, Valencia, 2017, pág. 389. 
Lo expuesto provoca que no se consiga el elemento de seguridad necesario del que sí se dispondría con la calificación como crédito contra la masa por mandato judicial y no por acuerdo entre las partes para aquellos supuestos en los que el concurso finalice con un convenio entre los acreedores.

Asimismo, la reforma introdujo en el propio 84.2.11 LC la consideración como crédito contra la masa del $50 \%$ de los créditos que supongan nuevos ingresos de tesorería. Sin embargo, respecto a ello debe indicarse, por un lado el importante riesgo que supone para los financiadores ver únicamente protegido el $50 \%$ de su crédito, siendo relegado el restante $50 \%$ a la consideración de crédito con privilegio general (91.6. $\left.{ }^{\circ} \mathrm{LC}\right)$, y por el otro, que esta calificación del crédito sólo es posible si la financiación ha sido concedida en el marco de un acuerdo de refinanciación, el cual es previo a la declaración de concurso (71 bis LC) constituyendo una forma de financiación preconcursal.

Efectuadas estas precisiones, en las siguientes líneas pretendemos realizar una aproximación a los mecanismos con los que cuentan actualmente los deudores en situación de insolvencia declarada para conseguir nuevos ingresos y establecer cuáles son los pasos que deberían seguirse por el legislador para facilitar la concesión de nueva financiación.

\section{ENTIDADES DE CRÉDITO Y FINANCIACIÓN POSTCONCURSAL}

Hablar de financiación conlleva inexorablemente hacer referencia a las entidades crediticias. Y es que para que el deudor en concurso pueda continuar su actividad comercial precisa del apoyo de entidades de crédito que le proporcionen el músculo financiero que le permita asumir las obligaciones económicas que la continuación de referida actividad supone ${ }^{2}$.

Sólo a través de estos nuevos ingresos se generará el flujo de liquidez necesario que posibilite mantener en funcionamiento la actividad del deudor y que a su vez permita cumplir con los compromisos adquiridos ${ }^{3}$.

2 La Exposición de Motivos de la Ley 5/2015, de 27 de abril, de fomento de la financiación empresarial pone esta situación de manifiesto al destacar la tradicional dependencia de la financiación bancaria por parte de las empresas españolas, sobre todo de las de menor dimensión.

${ }^{3}$ La doctrina alemana ha distinguido dos tipos de créditos en relación a la nueva inyección de liquidez: Überbrückungskredit y Sanierungskredit. Los prime- 
Para que las entidades financieras proporcionen esta inyección de liquidez tan necesaria, éstas precisan seguridad, es decir, que ante la evidente situación de riesgo que constituye todo concurso de acreedores, se le garantice el cobro de un crédito que ha sido concedido en una situación de insolvencia ya declarada. Y esta seguridad en el cobro, en ausencia de bienes que pudieran asegurar la operación, sólo puede conseguirse a través del mecanismo utilizado para garantizar el cobro de los créditos contra la masa: la prededucibili$\mathrm{dad}^{4}$. Es por este motivo que debe privilegiarse el crédito de aquel acreedor que está dispuesto a otorgar financiación para que referido acuerdo salga adelante.

$Y$ es que no puede olvidarse que conceder financiación a una empresa insolvente y en el marco de un procedimiento judicial supone un importante factor de riesgo que debe ser compensado de algún modo por parte del ordenamiento jurídico para hacer atractiva para el prestamista este negocio jurídico ${ }^{5}$.

Como respuesta, el texto concursal prevé dos posibilidades para que el crédito concedido por la entidad financiera sea calificado como crédito contra la masa:

1. Considerarlo como un crédito que se genera en el ejercicio de la actividad profesional o empresarial del deudor (84.2.5. $\left.{ }^{\circ} \mathrm{LC}\right)$.

2. Considerarlo como un crédito que resulta de obligaciones válidamente contraídas durante el procedimiento por la Administración Concursal o por el concursado con la autorización de ésta (84.2.9. $\left.{ }^{\circ} \mathrm{LC}\right)$.

ros son aquellos que se conceden para salir de la situación de crisis y continuar con la actividad económica del deudor y los segundos son aquellos que se proporcionan en un momento posterior cuando se ha comprobado la viabilidad de la actividad y permiten recuperar la situación financiera. Vid. al respecto PALAO UCEDA, J., "La finalización del procedimiento iniciado por insolvencia inminente» La insolvencia inminente y el sistema concursal preventivo, Bosch, Barcelona, 2013, pág. 303.

${ }^{4}$ Respecto al término prededucibilidad vid. BELTRÁN, E., "Comentario art. 84» en ROJO, A., BELTRÁN, E., Comentario de la Ley Concursal, Civitas, Madrid, 2004, tomo I. pág. 1499 donde se explica que el uso de la expresión, aun no siendo utilizado en ningún precepto del texto concursal, viene a hacer referencia al hecho de que estos créditos se satisfacen con preferencia y al margen del procedimiento concursal, antes del reparto propiamente dicho.

5 En igual sentido vid. ALONSO LEDESMA, C., "Créditos contra la masa, comunicación, reconocimiento y clasificación de créditos», Revista de Derecho Concursal y Paraconcursal, n. ${ }^{\circ}$ 14, Primer semestre de 2011, pág. 41. 
Nos decantamos por la primera de las opciones ${ }^{6}$, es decir por la consideración de la financiación concedida al deudor tras la declaración de concurso como un crédito contra la masa que se genera en el ejercicio de su actividad (84.2.5. ${ }^{\circ}$ LC) y ello por lo siguiente:

1. Debe considerarse la inyección de financiación como un acto que busca que el deudor continúe con su actividad económica. Productos bancarios como las líneas de crédito están principalmente orientadas a que se pueda hacer frente a las obligaciones más inmediatas de la actividad empresarial y son muy habituales en el tráfico mercantil diario.

2. El acuerdo al que llegue el deudor con un tercero (ya sea entidad bancaria o no) no siempre necesita de autorización expresa por parte de la Administración Concursal —más allá del régimen de autorización genérica previsto en el artículo 44.2 LC, ausente en aquellos supuestos en los que el acto se produzca antes de la aceptación de la Administración Concursal, siempre que el mismo sea imprescindible para continuar la actividad y se ajuste a las condiciones normales del mercado-. De considerarlo como un crédito del 84.2.9..$^{\circ} \mathrm{LC}$, solo aquellos acuerdos de financiación en los que haya intervenido específicamente la Administración Concursal — como protagonista o con su autorización- serán considerados como crédito contra la masa ${ }^{7}$.

Independientemente de su concreta ubicación normativa, es pacífica la consideración como crédito contra la masa del crédito surgido por la inyección de liquidez efectuada durante la fase común del procedimiento. El problema que encontramos es que en determinados procedimientos la calificación del crédito concedido por el financiador postconcursal como un crédito contra la masa no genera el suficiente incentivo para que este posible nuevo acreedor acceda a otorgar tan necesaria liquidez, sino que se requiere algo más ${ }^{8}$ Y es que no

${ }^{6}$ Misma postura parece mantener AZNAR GINER, E., «Fresh Money, Acuerdo de Refinanciación y el Concurso de Acreedores»Refinanciaciones de deuda, Acuerdos Extrajudiciales de Pago y Concurso de Acreedores, Tirant Lo Blanch, Valencia, 2013, pág. 312.

${ }^{7}$ Quedándose fuera de esta categoría financiaciones propias de la actividad económica habitual como por ejemplo el recurso al pagaré.

8 Vid en este sentido, MÍNGUEZ PRIETO, R.; BUIL ALDANA, I., "Restricción crediticia, sistema financiero "en la sombra» y mercado distressed: una aproximación a sus principales aspectos regulatorios, legales y prácticos», Revista de Derecho del Mercado de Valores, n. ${ }^{\circ}$ 14, Sección Estudios, Primer semestre de 2014, quienes tras reconocer que la financiación postconcursal en la fase común es posible, critican el régimen de incentivos tan insuficiente que contiene nuestra regulación. 
es infrecuente aquella situación en la que el informe de la Administración Concursal contiene una excesiva proliferación de créditos contra la masa que desaconseje a los posibles inversores financiar la actividad del concursado ante la alta probabilidad de no recuperar su dinero.

Para remediarlo, existen dos opciones:

1. ${ }^{\text {a }}$ Otorgar una garantía que asegure el posterior cumplimiento del deudor (supuesto ideal pero altamente complicado como veremos a continuación, ya que es muy improbable que el deudor disponga de bienes inmuebles sin cargas susceptibles de ser utilizados para garantizar la operación, salvo que se acudiese a bienes previamente gravados).

2. ${ }^{a}$ Privilegiar estos nuevos créditos contra la masa por encima de los demás créditos contra la masa. Probablemente estemos ante la solución menos popular pero más realista. Y es que la única manera de que los eventuales financiadores estén dispuestos a conceder liquidez al deudor que no puede ofrecer una garantía real es por medio de un privilegio dentro de los propios créditos contra la masa que le aseguren con un alto grado de certidumbre el cobro de su crédito. En el estado normativo actual, el crédito del financiador postconcursal sería abonado a su vencimiento conforme al artículo $84.3 \mathrm{LC}$ - salvo alteración de esta regla de pago por parte de la Administración Concursal- y con cargo a bienes no afectos a créditos con privilegio especial (154 LC). Lo que proponemos es blindar estos créditos para que gocen del mismo privilegio del que gozan los créditos administrativos - créditos tributarios y de la Seguridad Social- conforme veremos a continuación.

De igual forma, no puede obviarse la problemática ocasionada por la calificación del crédito del financiador cuando éste es otorgado en el marco de la fase de convenio. Referida problemática se ha venido suscitando cuando la financiación es concedida en aquellos supuestos en los que se ha aprobado judicialmente un convenio entre los acreedores. Efectivamente y como continuación a lo expuesto en el apartado introductorio, con base en el artículo 84.2.11. ${ }^{\circ}$ LC los préstamos concedidos por las entidades bancarias tras la apertura de la fase de convenio se considerarán créditos contra la masa si se cumplen dos condiciones:

1. ${ }^{\text {a }}$ Que el crédito sea concedido en el marco de un convenio.

2. ${ }^{\text {a }}$ Que finalmente se abra la fase de liquidación. 
De esta forma, sólo en aquel supuesto en el que la entidad bancaria acceda a conceder financiación al deudor en el marco de un acuerdo con el resto de acreedores y referido acuerdo posteriormente fracasara, ésta tendrá la seguridad del cobro de sus créditos al ver calificados los mismos como créditos contra la masa por Ley.

Si la financiación se concede mediante acuerdo pero no hay liquidación, se produce la remisión al artículo 100.5 LC, el cual indica que los créditos que se concedan al concursado para financiar el plan de viabilidad se satisfarán en los términos fijados en el convenio, expresión que introduce la posibilidad de que los acreedores pacten otra forma de satisfacción de estos créditos 9 .

$\mathrm{Y}$ es que como se ha hecho referencia, uno de los elementos determinantes de este tipo de créditos y lo que provoca precisamente su seguridad es la prededucibilidad en el cobro, de tal forma que si se altera vía convenio la forma de satisfacción de los mismos, se modificaría consecuentemente su calificación, ya que sin el elemento prededucible, el crédito contra la masa pierde su principal razón de ser.

La situación actual en la que se encuentra por tanto la normativa concursal no genera ningún incentivo a las entidades bancarias para que concedan financiación en el marco de un convenio, ya que sólo el fracaso de referido acuerdo y la posterior apertura de la fase de liquidación le otorgarán cierta seguridad en el cobro con la calificación de su crédito como crédito contra la masa. Por ello, concebimos como una gran grieta de nuestro sistema concursal no asegurar vía Ley la consideración del crédito concedido en fase de convenio como crédito contra la masa, fomentando de esta forma la entrada de dinero en la actividad económica del deudor.

${ }_{9}$ Respecto a este particular, resulta de interés destacar la Sentencia del Juzgado de lo Mercantil n. ${ }^{\circ}$ 2, de Bilbao, de 8 de junio de 2010 (Proc. 974/2009), en la que se realiza una interpretación finalista en relación a la consideración de créditos contra la masa que deben tener los generados en el periodo intermedio que transcurre desde la aprobación del convenio hasta la apertura de la fase de liquidación por incumplimiento, en los siguientes términos: En consecuencia, debe concluirse que no puede mantenerse la mera concursalidad de los créditos por aplicación literal de los preceptos legales referidos, puesto que, por un lado, es evidente que los acreedores necesitan un estímulo para seguir prestando sus créditos, sin los cuales el convenio fracasará; o, cuanto menos, no es recomendable desanimarles al respecto (con la perspectiva concursal de sus créditos). 


\section{ADMINISTRACIÓN CONCURSAL Y FINANCIACIÓN POSTCONCURSAL}

La Administración Concursal ostenta dos importantes facultades para influir en la financiación postconcursal que puede recibir el deudor.

La primera de ellas se encuentra regulada en el artículo 40 LC y es la relativa a su intervención respecto al ejercicio por el deudor de sus facultades patrimoniales, siendo ésta quien autoriza sus operaciones en el caso de que el mismo tenga sus facultades meramente intervenidas ( $40.1 \mathrm{LC}$ ) o quien le sustituye en la toma de decisiones cuando a éste le han sido suspendidas sus facultades (40.2 LC). Su importancia deriva de que los acuerdos que se adopten para conseguir financiación por parte del deudor son actos patrimoniales autorizables por la Administración Concursal en los casos de intervención de facultades o incluso suscritos por ésta en aquellos procedimientos en los que las facultades patrimoniales del deudor se encuentren suspendidas. Ello otorga un papel fundamental al órgano técnico concursal de cara a la inyección de financiación que puede recibir el deudor.

Lo expuesto debe entenderse necesariamente en consonancia con el artículo 43 LC en relación a la conservación y administración de la masa activa, precepto que en su apartado 3 permite a la Administración Concursal realizar - o autorizar - los actos de disposición que considere indispensables para garantizar la viabilidad de la empresa o las necesidades de tesorería que exija la continuidad del concurso - comunicando inmediatamente al Juez del concurso referida actuación y justificando su necesidad ${ }^{10}$ - Entre estas actuaciones consideramos que se encuentra la de conseguir financiación que permita la entrada de capital liquido en la actividad del deudor, ya que la nueva financiación es en la mayoría de ocasiones como decimos indispensable para que el deudor pueda hacer frente a las ne-

10 Vid CORDÓN MORENO, F., "Comentario art. 43», en CORDÓN MORENO, F. (dir.), Comentarios a la Ley Concursal, Thomson Reuters Aranzadi, Navarra, 2010, 2. ${ }^{a}$ edición, tomo I, pág.515, donde se señala que en definitiva se deja en manos de la Administración Concursal determinar cuál es el ámbito de las operaciones propias del tráfico o giro de la actividad. No obstante lo expuesto por el profesor CORDÓN MORENO, tras la reforma de este precepto por la Ley 38/2011, se estableció que aun en ese caso debía comunicarse inmediatamente al Juez del concurso los actos realizados. Sin embargo, debe precisarse que igualmente la decisión respecto a si el acto en concreto es indispensable para garantizar la viabilidad de la empresa o las necesidades de tesorería que exija el concurso sigue siendo competencia exclusiva de la Administración Concursal, por lo tanto es este órgano quien en última instancia decide si el acto debe estar sometido o no a autorización judicial. 
cesidades propias de la continuación de su operativa comercial, así como a los costes que todo procedimiento concursal origina.

Concebir las operaciones que consigan la entrada de financiación al patrimonio del concursado como un acto que no necesita autorización judicial es fundamental para el procedimiento, ya que evita la puesta en funcionamiento del régimen de autorización judicial contemplado en el artículo 188 LC, el cual prevé el traslado entre las partes de la solicitud de autorización, con el consiguiente plazo para alegaciones y resolución, demora incompatible con las características de premura y celeridad propias de la financiación postconcursal, que podrían frustrar la oportunidad que se presenta.

La segunda de las facultades se la confiere el artículo 84.3 LC y consiste en la posibilidad de alterar la regla de pago de los créditos contra la masa a su vencimiento cuando lo considere conveniente para el interés del concurso ${ }^{11}$. Referida facultad genera cierta inquietud para aquellos titulares de créditos que supongan nuevos ingresos de tesorería, puesto que se permite a la Administración Concursal alterar la regla de pago y postergar éste en beneficio de otros créditos contra la masa.

Meritada modificación del orden de pago no encuentra limitación al no encontrarse los créditos concedidos por estos nuevos ingresos de tesorería dentro de las excepciones a la postergación que se contempla en el artículo 84.3 LC, lugar reservado para los créditos de los trabajadores, créditos alimenticios y créditos tributarios y de la Seguridad Social.

Esta importante facultad de la Administración Concursal provoca un importante debilitamiento de la figura del financiador postconcursal, al verse condicionado por la decisión que pudiese adoptar ésta respecto a la posible postergación de su crédito bajo la amplia interpretación que el concepto «interés del concurso» ampara.

Como solución a esta situación, se plantean dos opciones: o bien limitar este poder de la Administración Concursal para que ello no suponga un obstáculo que frene la entrada de tan necesaria nueva financiación ${ }^{12}$ o bien incluir la entrada de este flujo de dinero nuevo

${ }^{11}$ El Tribunal Supremo ha insistido en que la alteración no queda al arbitrio de la Administración Concursal, sino que debe venir justificada por el interés del concurso. Vid. STS de 22 de julio de 2015 (Recurso 2003/2013).

12 Prueba de esta importancia es la expresión en inglés: "cash is king» (el dinero en efectivo es el rey) utilizada para destacar la importancia en una empresa del flujo de efectivo a corto plazo. 
dentro de las excepciones de postergación que el propio 84.3 LC prevé.

Ambas soluciones plantean sus problemas. Limitar la facultad de la Administración Concursal conllevaría redefinir el concepto de interés del concurso y atacar la profesionalidad de la figura, la cual ostenta entre sus funciones la posibilidad discrecional de decidir, con el correspondiente amparo judicial, que es lo mejor para el interés del concurso, es decir, para el interés de los acreedores. Sin embargo, incluir la nueva financiación dentro de una excepción donde se encuentran créditos que garantizan necesidades tan primordiales como son los salarios de los trabajadores o en los supuestos de concursos de una persona física el derecho de alimentos del propio deudor y su familia, podría conllevar debilitar consecuentemente al resto de figuras incluidas en esta excepción.

En una consideración de conjunto de lo expuesto, entendemos que la entrada de los créditos concedidos en virtud de nueva financiación dentro de la excepción que contempla el artículo 84.3 supone una intromisión menos gravosa para el procedimiento que lo que conllevaría limitar la facultad de interpretación del concepto «interés del concurso». Para ello, sería necesario que los créditos tributarios y de la Seguridad Social saliesen de referida excepción, ocupando su lugar los créditos derivados de la nueva financiación concedida al deudor en el marco del procedimiento. Ello armonizaría una interpretación del precepto conforme a parámetros de necesidad, marcados por la importancia de garantizar los créditos de los trabajadores, los alimentos del deudor (en su caso) y la nueva financiación tan imprescindible para que la actividad del concursado pueda continuar y satisfacer así el interés de los acreedores, parámetro que no se cumple incluyendo en este apartado los créditos tributarios y de la Seguridad Social.

Por último y en caso de insuficiencia de masa activa, acudiríamos al orden de prelación del artículo 176 bis LC, el cual no dibuja un escenario demasiado alentador para el financiador postconcursal al quedar su crédito contra la masa relegado al quinto lugar como «los demás créditos contra la masa» al carecer de encaje en el resto de apartados que prevé el precepto ${ }^{13}$.

13 Vid en igual sentido PULGAR EZQUERRA, J. «Fresh Money y financiación de empresas en crisis en la Ley 38/2011» en Revista de Derecho Concursal y Paraconcursal, n. ${ }^{\circ}$ 16, Sección Estudios, Primer semestre de 2012, pág. 67. 


\section{ACREEDORES Y FINANCIACIÓN POSTCONCURSAL}

Los acreedores son el elemento más importante que tiene el deudor concursado y al que más debe cuidar, ya que en interés de ellos se tramita el procedimiento concursal y es a ellos a quienes corresponde la última palabra respecto a un posible acuerdo que evite la liquidación del patrimonio intervenido judicialmente y el consiguiente fin de la actividad económica del deudor.

Pero la importancia de los acreedores no solo radica en los posibles acuerdos que puedan suscribir con el concursado para financiar su actividad comercial, sino que también son muy significativos aquellos pactos que pueden alcanzar con terceros ajenos al procedimiento en cuanto a su posición en el concurso.

En relación a ello, el artículo 122 LC en su redacción inicial impedía el derecho a voto en la Junta de Acreedores de aquellos acreedores que hubieran adquirido su crédito por cualquier tipo de negocio jurídico tras la declaración de concurso. Referido artículo fue objeto de reforma por el Real Decreto Ley 11/2014, de 5 de septiembre, de medidas urgentes en materia concursal, disposición legal que eliminó referida limitación ${ }^{14}$.

La modificación del precepto supuso un claro cambio de rumbo por parte del legislador español de cara a fomentar la existencia de un mercado de crédito entre los acreedores y terceros que permitiese la entrada en el procedimiento concursal de nueva financiación, además de otorgar a los acreedores una alternativa para salvar parte de su deuda ${ }^{15}$.

${ }^{14} \mathrm{Vid}$. la crítica que efectúa a esta limitación NÚNEZZ-LAGOS BURGUERA, A. «Restricciones en el mercado español de deuda concursal: el artículo 122.1.2 de la Ley Concursal» en Revista de Derecho Concursal y Paraconcursal, n. ${ }^{\circ} 7$, Sección Varia, Segundo semestre de 2007, pág. 129 quien con anterioridad a la reforma del precepto ya advertía que las actuaciones que el legislador pretendía evitar con la limitación del voto ya no eran posibles en la nueva regulación introducida por la Ley Concursal.

${ }^{15}$ Vid la exposición de motivos del Real Decreto-ley 11/2014, de 5 de septiembre, de medidas urgentes en materia concursal, en cuyo apartado III se hace referencia al cambio de normativa y a los beneficios que referida modificación conlleva para el deudor y los acreedores. Según se explica en el propio texto normativo, con esta limitación se pretendía evitar fraudes (entendemos que fundamentalmente se intentaba evitar prácticas oportunistas que impidiesen alcanzar un acuerdo entre acreedores). Sin embargo, el legislador rectifica al entender que ningún fraude puede existir en el simple hecho de comprar un crédito por un precio inferior, ya que es algo propio de la actividad económica de mercado. Interesa destacar como el propio legislador señala como efecto beneficioso de la liberalización del mercado de crédito en el seno del concurso la obtención de liquidez para los propios acreedores al poder recuperar parte de su inversión sin tener que esperar a la fase de liquidación. 
De esta forma, el acreedor inicial que vende su crédito, aun perdiendo parte del mismo debido al descuento lógico que el importe de venta conlleva como consecuencia del riesgo asociado al cobro, consigue recuperar parte de una deuda en ocasiones de muy dudosa posibilidad de reembolso y respirar económicamente en una situación que ha podido tener consecuencias incluso para su propia economía.

El nuevo acreedor que adquiere un crédito una vez iniciado el concurso, se coloca en la misma situación que el resto en la Junta de acreedores, teniendo de esta forma los mismos derechos y posibilidades. Su incentivo en la compra reside por un lado en la posibilidad de recuperar en el seno del concurso un mayor importe que el abonado previamente por el crédito al acreedor originario mediante el descuento asociado al riesgo, y por otro lado en la posibilidad de aprovechar una oportunidad de negocio mediante la inversión en el patrimonio del concursado.

Detrás de todo ello subyace el hecho de que se otorga flexibilidad en la titularidad de las deudas del deudor, facilitando la entrada de nuevos acreedores dispuestos — con la información pertinente- a inyectar liquidez al patrimonio para recuperar su inversión en un espacio temporal prolongado ${ }^{16}$.

\section{GARANTÍAS REALES Y FINANCIACIÓN POSTCONCURSAL}

Los bienes de los que es titular el deudor en el momento de la declaración del concurso constituyen el principal reclamo para que los inversores decidan inyectar dinero al patrimonio intervenido judicialmente. En la actual situación, nuestra normativa únicamente puede ofrecer en el mejor de los casos la calificación de su crédito como crédito contra la masa, circunstancia que se está demostrando como no suficiente, dada la escasa influencia que ello está teniendo en la concesión de financiación tras la apertura del concurso.

Y es que la posibilidad de constituir una garantía real sobre alguno de los bienes del deudor con el consiguiente privilegio que

${ }^{16}$ Así vid. MINGUEZ PRIETO, R.; BUIL ALDANA, I., "Restricción crediticia, sistema financiero "en la sombra» y mercado distressed: una aproximación a sus principales aspectos regulatorios, legales y prácticos» en Revista de Derecho del Mercado de Valores, n. ${ }^{\circ}$ 14, Sección Estudios, Primer semestre de 2014 en el que se expone los perjuicios que la prohibición hasta 2012 existente suponía para el propio concursado al ver eliminada la posibilidad de acceder a potenciales fuentes de financiación. 
ello otorga —el privilegio de ejecución separada previsto en el artículo 155 LC - supone un aliciente muy importante en la búsqueda de «dinero nuevo». Si el deudor dispone de bienes sobre los que no pese gravamen previo alguno, los mismos servirán para garantizar la deuda del nuevo financiador, a quien se le asegurará el pago de su crédito con la ejecución del bien sobre el que se ha constituido la garantía.

Sin embargo, esta idílica situación no es la habitual, ya que en la mayoría de las ocasiones cuando el concurso es declarado, la totalidad de los bienes del deudor - o al menos los más importantes- se encuentran gravados para responder de préstamos que precisamente buscaban evitar la situación de insolvencia o incluso con embargos consecuencia de la incapacidad para hacer frente a las obligaciones contraídas.

Esta circunstancia produce que deban idearse otros mecanismos que permitan garantizar la entrada de nueva financiación en el procedimiento, ya que es precisamente la ausencia de referidos mecanismos de incentivo lo que provoca que la solución del concurso sea casi irremediablemente la liquidación ${ }^{17}$.

Todo ello provoca que debamos efectuar una reflexión respecto a la introducción en nuestra legislación de la posibilidad de constituir garantías sobre activos del deudor ya gravados. Para ello, previamente debería llegarse a un acuerdo con el acreedor preexistente por medio del cual éste consienta ceder el primer lugar en el orden de cargas concurrentes respecto a un determinado bien del deudor. Este acreedor continuaría con su carga inscrita, habiendo cedido su posición privilegiada a un tercero, quien cobraría con carácter previo a éste de la ejecución del bien en el supuesto de impago del deudor, facilitando de esta forma la entrada de nueva financiación en el procedimiento.

$17 \mathrm{Vid}$. lo establecido respecto a la constitución de garantías reales sobre bienes del deudor (tanto libres como gravados) en ASENSIO PASCUAL, C., « ¿Es la financiación postconcursal la gran olvidada de nuestro legislador?», en Revista de Derecho Concursal y Paraconcursal, n. ${ }^{\circ}$ 24, Sección Comunicaciones, Primer semestre de 2016 quien basándose en las recomendaciones de la Comisión de las Naciones Unidas para el Derecho Mercantil Internacional (UNCINTRAL) señala que incluso cuando el acreedor garantizado ya existente no prestara su consentimiento, el Tribunal podrá autorizar la constitución de una garantía real que goce de prelación sobre la preexistente siempre que se notifique al acreedor, el deudor no pueda obtener financiación de otra manera y se protejan los intereses del acreedor garantizado ya existente. 


\section{LA FINANCIACIÓN POSTCONCURSAL EN EL DERECHO COMPARADO}

A diferencia de nuestro país, en el resto de estados de nuestro entorno la financiación postconcursal sí ha gozado de una mayor atención por parte del legislador.

Pero es sin duda la legislación norteamericana ${ }^{18}$, con su propia concepción de la insolvencia, la que más incentivos contiene a la hora de fomentar la entrada de liquidez en el patrimonio del deudor insolvente. El artículo 364 del Código de Insolvencia Norteamericano (conocido como Bankruptcy Code) es el encargado de establecer las diferentes posibilidades que se otorgan a acreedores y deudor para conseguir financiación. Entre los incentivos más importantes destacan el hecho de poder constituir gravámenes de segundo nivel (o garantías junior) sobre bienes del deudor ya gravados previamente y sobre todo la posibilidad de conceder como garantía a los nuevos financiadores los activos del deudor con independencia de que sobre éstos exista ya un gravamen previo. Para ello es requisito indispensable tener adecuadamente protegidos a los acreedores cuya obligación se encontraba garantizada con cargo a referidos activos con carácter previo a los nuevos financiadores.

\section{CONCLUSIONES}

La entrada de nueva financiación en el procedimiento concursal es una cuestión tan necesaria como problemática.

Necesaria porque en la situación de insolvencia en la que se encuentra el deudor, disponer de fondos para hacer frente a las obligaciones más inmediatas proporciona a éste la posibilidad de continuar con su actividad económica y que sea precisamente su actividad la que le permita salir de la delicada situación económica en la que está, pudiendo cumplir así con sus obligaciones, abandonando cuanto antes el hecho de ver su patrimonio intervenido judicialmente.

${ }_{18}$ Nos remitimos al completo estudio llevado a cabo por BUIL ALDANA, I., «La financiación de empresas en situación concursal: una visión española basada en el debtor-in-possession financing del sistema norteamericano de insolvencias», Revista de Derecho Concursal y Paraconcursal, n. ${ }^{\circ}$ 14, Sección Derecho Comparado, Primer semestre de 2011, pág. 373 en el que además se hace referencia al nacimiento y la consolidación de la financiación postconcursal en EE.UU. 
Problemática porque para conseguir la inyección económica en el patrimonio del deudor, es necesario ofrecer al nuevo acreedor incentivos que le lleven a querer invertir, incentivos que sólo pueden conseguirse a costa de los acreedores iniciales, quienes tienen que soportar un perjuicio en su propia posición primigenia para garantizar la entrada de nuevos ingresos en el patrimonio del deudor que le permitan a éste continuar con su actividad económica y poder hacer frente así al pago de los créditos del resto de acreedores.

$\mathrm{Y}$ es que el hecho de que nuestro sistema carezca del adecuado mecanismo de financiación postconcursal provoca que no se le otorgue al deudor en concurso el elemento necesario para continuar su actividad, lo que genera no sólo que el mismo se vea abocado a la liquidación de su patrimonio, sino también el propio deterioro de referido patrimonio al carecer la masa de fondos para el mínimo mantenimiento de los activos propiedad del deudor concursado.

Como solución a esta situación hemos apuntado fundamentalmente dos en el presente trabajo: por un lado modificar la consideración de determinados créditos como créditos contra la masa para que su pago no pueda ser postergado por la Administración Concursal, reforzando de este modo la seguridad en el cobro de los nuevos créditos que conceden financiación al deudor - eliminando de este privilegio a los créditos tributarios y de la Seguridad Social-y por el otro la posibilidad de introducir en nuestra legislación la constitución de garantías sobre activos del deudor ya gravados para garantizar los nuevos créditos derivados de las aportaciones dinerarias, previo acuerdo con el acreedor titular de referida garantía.

Debemos conseguir implantar en nuestro ordenamiento una situación de equilibrio entre los nuevos acreedores dispuestos a otorgar capital con los acreedores iniciales que han venido constituyendo la comunidad de pérdidas del deudor, puesto que en muchas ocasiones es la entrada de estos inversores y el capital que aportan a la actividad comercial del concursado la que posibilita que los acreedores primigenios pueden ver satisfechos sus créditos. 